\title{
Impact of Scene Decorrelation on Geosynchronous SAR Data Focusing
}

\author{
Andrea Recchia, Andrea Monti Guarnieri, Member, IEEE, Antoni Broquetas, Member, IEEE, and Antonio Leanza
}

\begin{abstract}
We discuss the effects of the clutter on geosynchronous SAR systems exploiting long integration times (from minutes to hours) to counteract for two-way propagation losses and increase azimuth resolution. Only stable targets will be correctly focused whereas unstable targets will spread their energy along azimuth direction. We derive here a generic model for the spreading of the clutter energy based on the power spectral density of the clutter itself. We then assume the Billingsley Intrinsic Clutter Motion model, representing the clutter power spectrum as an exponential decay, and derive the expected GEOSAR signal-toclutter ratio. We also provide some results from a Ground Based RADAR experiment aimed at assessing the long-term clutter statistics for different scenarios to complement the Internal Clutter Motion model, mainly derived for windblown trees. Finally, we discuss the expected performances of two GEOSAR systems with different acquisition geometries.
\end{abstract}

Index Terms-Focusing, Geosynchronous Synthetic Aperture Radar (GEOSAR), scene decorrelation, wind-blown clutter.

\section{INTRODUCTION}

A SYNTHETIC aperture radar (SAR) placed in geosynchronous orbit (GEOSAR) was first proposed at the end of the 1970s [1], [2]. Since then, several GEOSAR systems have been proposed but never implemented due to both technological constraints [3] and the presence of decorrelation sources impacting on the quality of the focused images [4]. Nevertheless, the growing interest in GEOSAR systems is justified by their potential applications. In particular, the daily revisit could enable the near real time monitoring of geophysical phenomena with time scales much faster than those currently observed with Low Earth Orbit SAR (LEOSAR) constellations.

GEOSAR systems exploit long integration times to increase resolution and compensate spread losses, making the scene response during the dwell time nonstable. From one side the Atmospheric Phase Screen (APS), if not properly compensated, could prevent data focusing when integration time extends from several minutes to hours [5]-[7]. On the other hand the response of the targets itself can change during the dwell time. The energy of nonstable targets, after focusing, will spread along

Manuscript received October 22, 2014; revised March 18, 2015 and July 17, 2015; accepted August 14, 2015. Date of publication October 30, 2015; date of current version February 24, 2016.

A. Recchia, A. Monti Guarnieri, and A. Leanza are with the Dipartimento di Elettronica, Informazione e Bioingegneria, Politecnico di Milano, 20133 Milano, Italy (e-mail: andrea.recchia@aresys.it).

A. Broquetas is with Universitat Politecnica de Catalunya, 08034 Barcelona, Spain.

Color versions of one or more of the figures in this paper are available online at http://ieeexplore.iee. org.

Digital Object Identifier 10.1109/TGRS.2015.2486385 azimuth direction affecting the signal-to-clutter ratio (SCR) even for very stable targets such as urban and rocky areas [4].

In the present paper we neglect atmospheric effects and concentrate on the impact of the clutter decorrelation on a GEOSAR system. For short integration time SAR systems $(<1 \mathrm{~s})$ the clutter is assumed stationary within the dwell time and, after focusing, impairs the detection of the targets of interest. Unlike thermal noise, the SCR cannot be improved by increasing the transmitted power but only enhancing the system resolution. Ulaby and Dobson experimentally derived models for the characterization of the clutter in terms of normalized radar cross section $\left(\sigma^{0}\right)$ for different classes of terrain at different grazing angles [8].

The increase of the integration time and the reduction of the wavelength in modern SAR systems, aimed at improving resolution like in Spotlight SAR [9], pose the problem of nonstationary clutter, which also affects applications like moving target indication [10], [11]. For this reason, nonstationary clutter has been widely investigated by Billingsley, who introduced the Internal Clutter Motion (ICM) model, experimentally derived for windblown trees at different bands (from $\mathrm{L}$ to $\mathrm{X}$ ) [12]. The ICM model is a good approximation of the nonstationary clutter spectrum for observation times up to $1 \mathrm{~min}$. The validity of the ICM model has never been proven for longer observation times like those exploited by a GEOSAR system, which can extend to hours [13], [14].

The aim of this paper is twofold. On one hand we introduce a theoretical model for the evaluation of the expected SCR in a generic GEOSAR system. The SCR model explicitly includes the effects of nonstationary clutter, always neglected for standard LEOSAR systems. The new performance models can be exploited for the design of future GEOSAR missions. On the other hand we provide a preliminary assessment of the ICM model validity for long integration times exploiting a set of ground-based RADAR acquisitions at Ku band. The analysis shows that the ICM model is not totally valid for long integration times, at least in terms of the values of the model parameters indicated by Billingsley. In any case further extensive GB RADAR campaigns should be carried out to provide a meaningful statistical characterization of the nonstationary clutter over long observation times and for different classes of targets.

The paper is organized as follows. In Section II we introduce the GEOSAR concept and define the received and focused signal models. In Section III we provide a statistical description of the nonstationary clutter as a Brownian motion, which can be directly related to the Billingsley ICM model. In Section IV we derive the theoretical expressions for the SCR in a generic GEOSAR system. Finally, in Section V we evaluate the SCR 


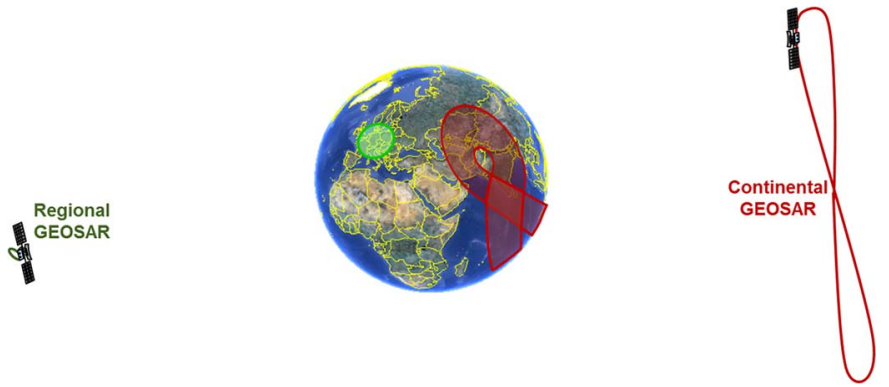

Fig. 1. Acquisition geometry and ground coverage of the continental (red) and regional (green) geosynchronous SAR systems.

TABLE I

Comparison Between Main System Parameters OF CONTINENTAL AND TLCOMP SYSTEMS

\begin{tabular}{c|c|c}
\hline System & Continental & TLCOMP \\
\hline $\begin{array}{c}\text { Orbit Inclination } \\
\text { Antenna } \\
\text { diameter } \\
\text { Average } \\
\text { Transmitted } \\
\text { Power } \\
\text { PRF }\end{array}$ & 50 & Close to 0 \\
Velocity @ \\
Equator \\
$\begin{array}{c}\text { Integration time } \\
\text { Atmosphere } \\
\text { Revisit time }\end{array}$ & $15-30 \mathrm{~m}$ & $400 \mathrm{~W}$ \\
Implementation & $1-2$ minutes & $50 \mathrm{~Hz}$ \\
& Almost Frozen & $\begin{array}{c}15 \mathrm{~m} / \mathrm{s} \\
\text { minutes - } 8 \text { hours } \\
\text { Censed - To Be } \\
\text { Compensated } \\
12 \text { hours }\end{array}$ \\
\hline
\end{tabular}

expressions for two different GEOSAR concepts, assessing their robustness in front of the scene decorrelation.

\section{GEOSAR CONCEPTS AND SignAL Models}

\section{A. GEOSAR Concepts}

Two main GEOSAR concepts have been proposed in literature. The first one achieves continental coverage by means of a significant orbit inclination [2]. Integration time in the order of minutes, high power, and quite large antennas are exploited to counteract for the spread losses. According to [3] road-map, such system would require 2020 technologies to be implemented.

A totally different concept, first proposed in [15], is based on a negligible orbit inclination, very long integration times, and reduced requirements in terms of power and antenna size, making it suited to be hosted as an additional payload on a commercial telecommunication satellite (TeLecom COMPatible concept).

Fig. 1 compares the two GEOSAR concepts in terms of orbit (in an Earth Fixed Reference System) and ground coverage. The continental system would allow to monitor a very large areasay from Equator to midlatitudes of both hemispheres - in a non continuous way. On the contrary the reduced orbit extension of the TLCOMP system would allow the continuous monitoring — say a low-resolution image every $15 \mathrm{~min}$ - of a region located at midlatitude such as Central Europe.

Table I provides a comparison between the main system parameters of the GEOSAR concepts. The values for the con- tinental system were retrieved from [2] and [16] while for the TLCOMP concept we take as reference the system proposed in [17]. GEOSAR systems would be the natural complement to standard LEOSAR sensors, providing nearly real time monitoring of events such landslides, motion of glaciers and volcanoes, ground subsidence and building deformation in urban areas. Furthermore, TLCOMP sensitivity to the atmospheric delay could be exploited to generate Water Vapor maps over stable land surfaces, which would provide valuable information to Numerical Weather Prediction models.

\section{B. GEOSAR Acquired Signal Model}

The impulse response function (IRF) of a GEOSAR system after demodulation into base band and range matched filtering can be written, like for any other SAR, as

$h_{\mathrm{SAR}}(t, \tau ; \mathbf{P})=w(\tau ; \mathbf{P}) g\left[t-\frac{R(\tau ; \mathbf{P})}{2 c}\right] \exp \left[-j \frac{4 \pi}{\lambda} R(\tau ; \mathbf{P})\right]$

where $t$ is the fast time, $\tau$ is the slow time, $\mathbf{P}$ is the threedimensional target location and we have assumed a narrowband (monochromatic) system with wavelength $\lambda$. The first term $w(\tau ; \mathbf{P})$ is a weighting factor accounting for antenna pattern and propagation losses, the second term is the delayed version of the compressed pulse $g(t)$, and the last term is the slant range phase depending on the sensor-to-target distance $R(\tau ; \mathbf{P})$. The acquired range compressed data are the spatially variant convolution between the ground scene and the GEOSAR impulse response

$$
d(t, \tau)=\int_{L} s(\mathbf{P}, \tau) h_{\mathrm{SAR}}(t, \tau ; \mathbf{P}) d \mathbf{P}
$$

where $L$ represents the area illuminated by the antenna main lobe and $s(\mathbf{P}, \tau)$ is the ground scene, assumed both spatially and temporally (during the illumination time) variant. Note that the scene dependency on the azimuth time is usually neglected in standard LEOSAR systems due to the very short dwell time. We are interested in assessing the effects of the decorrelating clutter on the reconstructed scene, assumed spatially impulsive for simplicity

$$
s(\mathbf{P}, \tau)=u(\tau) \delta\left(\mathbf{P}-\mathbf{P}_{0}\right)
$$

where $u(\tau)$ is the time varying complex reflectivity of the scene. By substituting (3) in (2) we get the model of the GEOSAR received data for an impulsive decorrelating scene

$$
d(t, \tau)=u(\tau) h_{\mathrm{SAR}}\left(t, \tau ; \mathbf{P}_{0}\right)
$$

\section{GEOSAR Focused Signal Model}

We assume to reconstruct the imaged scene with the time domain back-projection algorithm [18]. The BP integral for a generic scene location can be written as

$$
\widehat{s}(\mathbf{P})=\int_{T} d\left(\frac{R(\tau ; \mathbf{P})}{2 c}, \tau\right) \exp \left[j \frac{4 \pi}{\lambda} R(\tau ; \mathbf{P})\right] d \tau
$$




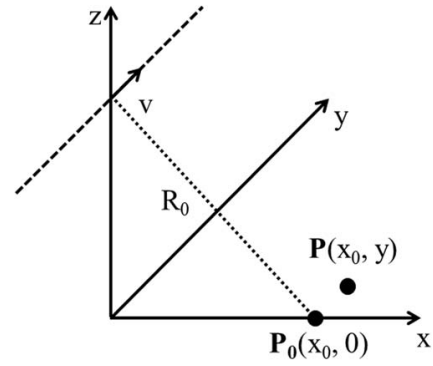

Fig. 2. Simplified geometry for azimuth IRF calculation.

where $T$ is the considered synthetic aperture time. Substituting (4) in (5) we get

$$
\begin{aligned}
\widehat{s}(\mathbf{P})=\int_{T} w\left(\tau ; \mathbf{P}_{0}\right) g & \left(\frac{\Delta R(\tau ; \mathbf{P})}{2 c}\right) u(\tau) \\
& \times \exp \left(-j \frac{4 \pi}{\lambda} \Delta R\left(\tau ; \mathbf{P}, \mathbf{P}_{0}\right)\right) d \tau
\end{aligned}
$$

where $\Delta R\left(\tau ; \mathbf{P}, \mathbf{P}_{0}\right)=R(\tau ; \mathbf{P})-R\left(\tau ; \mathbf{P}_{0}\right)$ is the range mismatch between the target location $\mathbf{P}_{0}$ and the reconstructed scene location $\mathbf{P}$. The above expression assumes that the motion of the target is confined within a range resolution cell so that no energy spreading along range occurs. This is reasonable for the natural targets (like cultivated fields or forests) we are interested in. Furthermore, considering the GEOSAR critical velocity defined in (12), the Doppler induced delay (or range shift) after pulse compression is small compared to the range resolution and has been neglected.

We now refer to the simplified geometry in Fig. 2 where the $y$-axis is aligned with azimuth direction. By approximating the range mismatch with a first-order Taylor expansion, we can write (6) as

$$
\widehat{s}\left(y ; R_{0}\right)=\int_{T} w\left(\tau, y ; R_{0}\right) u(\tau) \exp \left(-j 2 \pi \frac{2 y v}{R_{0} \lambda} \tau\right) d \tau
$$

where $R_{0}$ is the slant range, and we have assumed

$$
g\left(\frac{\Delta R(\tau ; \mathbf{P})}{2 c}\right) \approx 1
$$

The previous expression can be evaluated as a Fourier transform, and the reconstructed scene is

$$
\widehat{s}\left(y ; R_{0}\right)=W\left(\frac{2 v}{R_{0} \lambda} y\right) * U\left(\frac{2 v}{R_{0} \lambda} y\right)
$$

where $W$ is the GEOSAR system nominal IRF including acquisition and focusing, $U$ is the spectrum of the time-varying target reflectivity, and $*$ represents the convolution operator w.r.t. the azimuth coordinate $y$. We can rewrite (9) as

$$
\widehat{s}\left(f_{d}\right)=W\left(f_{d}\right) * U\left(f_{d}\right)
$$

where $f_{d}=(2 v / \lambda)\left(y / R_{0}\right)$ is the system Doppler frequency.

The result of the above convolution strongly depends on the spectral width of $W$ and $U$. In case $U$ is significantly narrower than $W$ we get the nominal IRF of the system. In case the width of $W$ and $U$ are of the same order of magnitude the result of the convolution cannot be easily modeled. This is the typical case of APS decorrelation [19]. Finally, in case $W$ is significantly narrower than $U$, we can assume that the system IRF is impulsive w.r.t. the clutter spectrum and we get

$$
\widehat{s}\left(f_{d}\right)=A \cdot U\left(f_{d}\right)
$$

where $A=\int W\left(f_{d}\right) d f_{d}$ is an amplitude term which will be neglected in the following derivation. The above assumption depends on the velocity of the imaged clutter compared with the Doppler bandwidth of the system. We define a critical clutter velocity $v_{c}$, representing the threshold above which the frozen clutter assumption is no longer valid

$$
v_{c}=v \frac{\rho_{a z}}{R_{0}}
$$

where $\rho_{a z}$ is the system azimuth resolution. Assuming an azimuth resolution of $10 \mathrm{~m}$ the critical velocity is around $10^{-6} \mathrm{~m} / \mathrm{s}$ for a regional GEOSAR system; around $10^{-3} \mathrm{~m} / \mathrm{s}$ for a continental GEOSAR system and around $0.1 \mathrm{~m} / \mathrm{s}$ for a LEOSAR system. From the above considerations every moving target in the scene can be assumed as decorrelating clutter for a GEOSAR system and modeled as in (11).

\section{Decorrelating Clutter}

\section{A. Windblown Clutter Model}

The content of a resolution cell during a long observation time can be modeled as a large set of independent elementary scatterers, some of them affected by Brownian motion. The radar echoes from such resolution cell can be expressed as

$$
u(\tau)=\sqrt{\alpha}+\sqrt{1-\alpha} \sum_{n} a_{n} \exp \left[-j \frac{4 \pi}{\lambda} x_{n}(\tau)\right]
$$

where $\alpha$ is a coefficient representing the fraction of power from the stable scatterers, $a_{n}$ is the complex reflectivity of each scatterer, and the stochastic variable $x_{n}(\tau)$ is the target displacement along the radar line of sight, modeled as a Brownian motion process whose value at a given time instant $\tau$ is distributed as $N\left(0, \sigma_{x, n}^{2} \tau\right)$. For simplicity we have assumed, with no impacts on the generality of the proposed model, that the stable targets component is real. The motion variance $\sigma_{x, n}^{2}$ depends on several factors including the scatterer nature (e.g., tree leaves are expected to have higher variance than tree branches), the season, the weather (especially local wind conditions), and even the time of the day. We assume here small values of $\sigma_{x, n}^{2}$ so that the displacement of a scatterer during the observation time is much lower than the resolution cell. This also means that for short time intervals the returns from the same scatterer can be correlated since the displacement values will be of the same order or even smaller than the wavelength.

The autocorrelation function of the sole time varying component of $u(\tau)$ is

$R_{u}\left(\xi ; a_{n}, x_{n}\right)=E\left[\sum_{n}\left|a_{n}\right|^{2} \int \exp \left[-j \frac{4 \pi}{\lambda} \Delta x_{n}(\xi)\right] d \tau\right]$ 
where we have neglected the constant $(1-\alpha)$ term and exploited the weak stationarity of the scatterers motion processes and their statistical independence. By exploiting the relationship $E[\exp (j \phi)]=\exp \left(-\sigma_{\phi}^{2} / 2\right)$ we can rewrite (14) as

$$
R_{u}\left(\xi ; a_{n}, \sigma_{x, n}^{2}\right)=\sum_{n}\left|a_{n}\right|^{2} \exp \left[-\left(\frac{4 \pi}{\lambda}\right)^{2} \frac{\sigma_{x, n}^{2}}{2} \xi\right] .
$$

By assuming that the complex reflectivity $a_{n}$ is a set of zero mean, independent random variables with $E\left[\left|a_{n}\right|^{2}\right]=P_{c}$, the autocorrelation function can be written as

$$
\begin{aligned}
R_{u}(\xi) & =E_{\left\{a_{n}\right\},\left\{\sigma_{x, n}^{2}\right\}}\left\{R_{u}\left(\xi ; a_{n}, \sigma_{x, n}^{2}\right)\right\} \\
& =P_{c} \int \exp \left[-\left(\frac{4 \pi}{\lambda}\right)^{2} \frac{\sigma_{x, n}^{2}}{2} \xi\right] f\left(\sigma_{x, n}^{2}\right) d \sigma_{x, n}^{2} n
\end{aligned}
$$

where $f\left(\sigma_{x, n}^{2}\right)$ is the probability density function of the Brownian motion variance. Finally, the clutter power spectrum can be written as

$$
\left|U\left(f_{d}\right)\right|^{2}=\alpha \delta\left(f_{d}\right)+(1-\alpha) \mathcal{F}\left\{R_{u}(\xi)\right\} .
$$

The previous expression is quite generic and can lead to different clutter power spectrum models such as Gaussian or Power Law. Billingsley showed both experimentally [12] and theoretically [20] that the power spectrum of windblown clutter can be approximated with an exponential decay at near grazing incidence and over short integration times. The clutter power spectrum according to Billingsley ICM model can be represented as

$$
\left|U\left(f_{d}\right)\right|^{2}=\frac{r}{r+1} \delta\left(f_{d}\right)+\frac{1}{r+1} S_{u}\left(f_{d}\right)
$$

where $r$ is the ratio of dc power to ac power in the spectrum and the spectral components of the time varying clutter show an exponential decay w.r.t. to the frequency

$$
S_{u}\left(f_{d}\right)=\frac{\beta \lambda}{4} \exp \left(-\frac{\beta \lambda}{2}\left|f_{d}\right|\right)
$$

where $\beta$ is the exponential shape parameter providing the decay rate and $\int\left|U\left(f_{d}\right)\right|^{2} d f_{d}=1$. Note that (18) and (19) can also be expressed in terms of Doppler velocity $v_{d}$ instead of Doppler frequency $f_{d}$ according to the well-known relationship $f_{d}=$ $2 v_{d} / \lambda$. Fig. 3 shows the typical triangular shape (in logarithmic scale) of the windblown clutter power spectrum. Three different components can be identified: a fast decorrelating component originated by the tails of the exponential spectrum folding back in base band due to the system sampling (scatterers with velocity much higher than $v_{c}$ ); a slow diffuse component with Doppler similar to the system bandwidth spreading its energy throughout the image (scatterers with velocity higher than $v_{c}$ ) and a mid-long-term coherent component which will result well focused or only slightly defocused (scatterers with velocity similar or even lower than $v_{c}$ ).

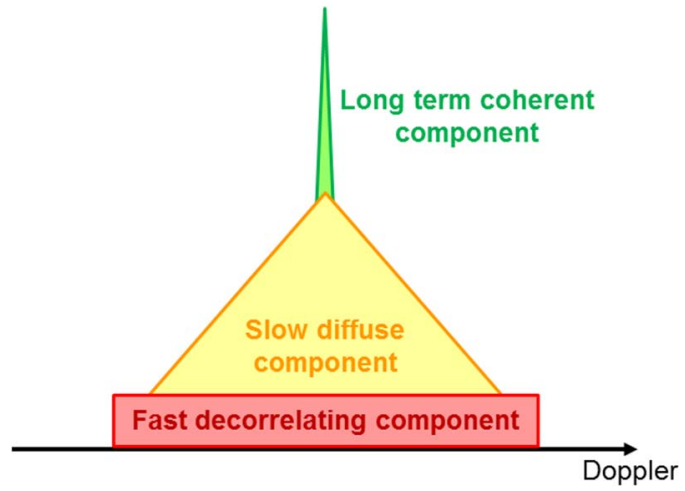

Fig. 3. Typical triangular shape (in logarithmic scale) of the clutter power spectrum according to Billingsley Intrinsic Clutter Motion Model. The fast decorrelating component is originated by the tails of the exponential spectrum folding back in base band due to the system sampling. The slow diffuse component fits the power law. The mid-long-term coherent component is the stable part.

TABLE II

TyPical Exponential Decay $\beta$ Values Measured UNDER DIFFERENT WIND CONDITIONS DURING BILLINGSLEY EXPERIMENTS [12]

\begin{tabular}{c|c|c}
\hline Wind & Wind speed & Decay Rate \\
\hline Light air & $1-3 \mathrm{~m} / \mathrm{s}$ & 12 \\
Breezy & $3-7 \mathrm{~m} / \mathrm{s}$ & 8 \\
Windy & $7-15 \mathrm{~m} / \mathrm{s}$ & 5.7 \\
\hline
\end{tabular}

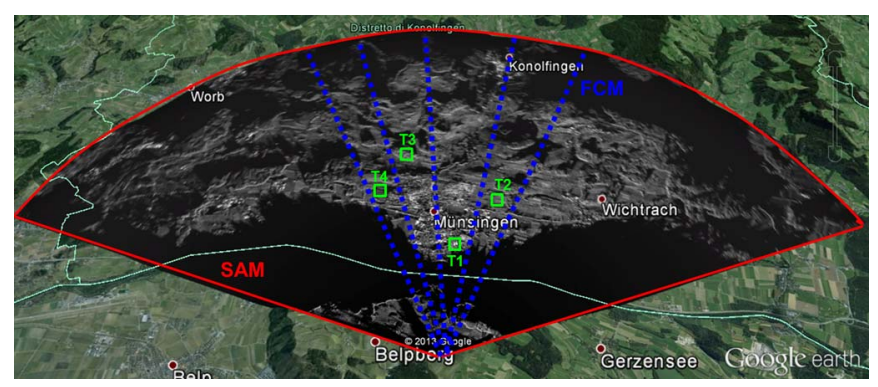

Fig. 4. Ground-based RADAR scenario (rural environment close to Bern) with SAM and FCM acquisition modes. The four targets indicated refer to the four spectra shown in Fig. 5. T1 is an urban target, T2 is a target from a cultivated area, T3 is from a wood, and T4 is on the edge between a small village and a field.

\section{B. Experimental Verification of Clutter Model}

The ICM model was experimentally derived for windblown trees at near grazing incidence angles over short integration times (lower than $1 \mathrm{~min}$ ). Measurements were performed at different bands from $\mathrm{L}$ to $\mathrm{X}$. From his measurement campaigns Billingsley provided an empirical relationship between the dc/ac ratio $r$, the carrier frequency $f_{c}[\mathrm{GHz}]$, and the wind speed $w[\mathrm{~m} / \mathrm{s}]$

$$
r=140.7 w^{-1.55} f_{c}^{-1.21} .
$$

For the exponential decay rate the values reported in Table II were measured under different wind conditions.

The ICM model is particularly suited for the GEOSAR continental system whose integration time is of the same order of magnitude. On the contrary for the regional system the 

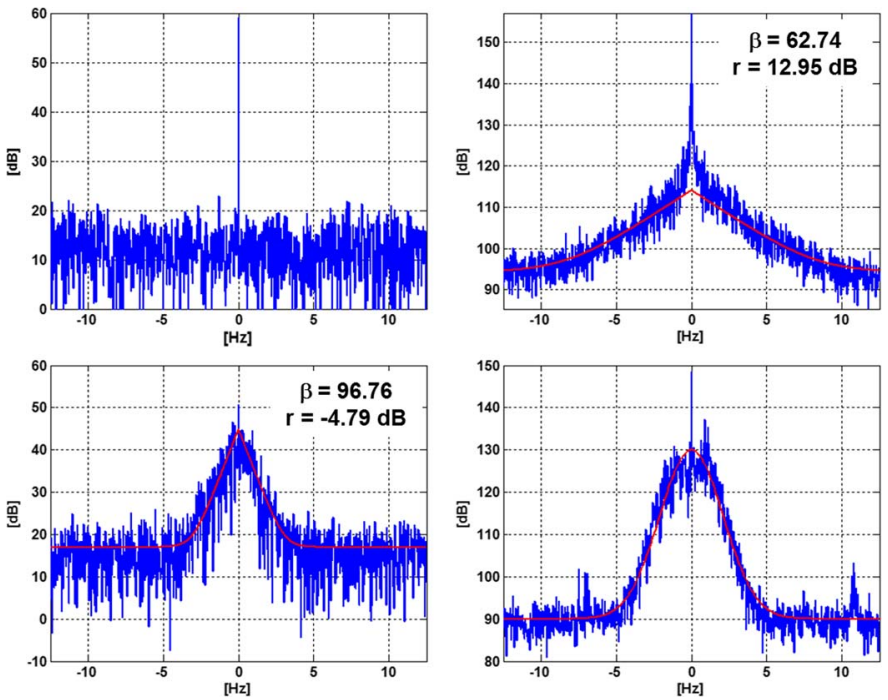

Fig. 5. Measured power spectrum (blue line) and corresponding Billingsley model fit (red line) for the four targets indicated in Fig. 4. The fitted model parameter values have been reported. The bottom left target was fitted with a Gaussian model instead of the ICM model. The wind speed during the considered acquisition campaign was low with a maximum speed of about $3 \mathrm{~m} / \mathrm{s}$.

integration time can be much longer (up to hours for high resolution applications). For this reason a ground-based RADAR experiment was designed to assess clutter long-term statistics. The experiment was performed with a portable RADAR interferometer working in $\mathrm{Ku}$ band (not included in Billingsley measurement campaigns) [21]. Fig. 4 shows a geocoded image of one of the monitored scenarios (rural environment close to Bern). The four targets indicated refer to the four spectra shown in Fig. 5. T1 is an urban target, T2 is a target from a cultivated area, T3 is from a wood, and T4 is located on the edge between a small village and a field. Two different acquisition modes are exploited:

- Scanning Atmosphere Mode (SAM): repeated scans of the same area with short temporal baseline (less than $3 \mathrm{~min}$ ) are performed. A stack of interferometric images is obtained for each measurement campaign.

- Fixed Clutter Mode (FCM): configuration based on Billingsley measurement campaigns. The instrument transmits and receives along a fixed direction for about one minute then changes the looking direction and the measurement is repeated. Acquisition parameters are quite different from Billingsley setup since we are mainly interested to the quasi-dc region (few $\mathrm{Hz}$ ) which is the most critical for a regional GEOSAR system. For this reason the acquisition pulse-repetition frequency (PRF) is lower than the one used during Billingsley campaigns.

FCM data have been exploited to derive the ICM model parameters for the different classes of imaged targets (ICM model mainly refers to forest) at $\mathrm{Ku}$ band. A minimum least square fit of Billingsley model was implemented [22]. Fig. 5 shows a few samples of the measured power spectra (blue line) and of the corresponding fits (red line) for the four targets indicated in Fig. 4. The top left target (T1) is a coherent urban target with a good dc component and a flat spectrum (white noise) outside the dc portion. The top right target (T2) is from a cultivated area with moderate $\mathrm{dc} / \mathrm{ac}$ ratio and quite high value of $\beta$. The bottom left target (T3) is from a wood with low dc/ac ratio and even higher value of $\beta$. Finally, the bottom right target (T4) is not well identified (it is located on the edge between a small village and a cultivated area) and shows an unexpected spectral shape which cannot be fitted with the ICM model but has a good agreement with the Gaussian function represented with the red line. Fig. 6 shows the distribution of the fitted $r$ parameter for tree acquisition performed in Bern on October 2013 (left) and May 2014 (center) and in Lugano on September 2014 (right). The red box indicates the range of expected values for windblown trees, calculated at $\mathrm{Ku}$ band according to (20) for wind speeds between $1 \mathrm{~m} / \mathrm{s}$ and $5 \mathrm{~m} / \mathrm{s}$. The measured values cover a much wider range from $-20 \mathrm{~dB}$ up to $40 \mathrm{~dB}$, due to the variety of targets observed in the scene and to the variability of the parameter during the 24-h campaigns. Fig. 7 shows the evolution of $r$ parameter versus time of the day for the same acquisitions considered in Fig. 6 (Blue: Bern November; red: Bern May; green: Lugano September). The dc/ac trends were realigned over a common time axis spanning $24 \mathrm{~h}$ from the midnight. The staring time of each 24-h campaign is indicated by the corresponding arrow. A day/night trend can be noticed with a maximum of dc/ac ratio during the night and a minimum during the day, in particular for the November acquisition close to Bern.

The data from SAM acquisitions, after APS compensation, are exploited to retrieve the clutter long-term statistics. APS estimation was performed through the coherent processing of a stack of interferometric images to identify Permanent Scatterers as described in [23]. Of course a residual APS variation, in particular over daytime images, could impact on the derived clutter statistics. This problem will also affect the GEOSAR systems, in particular the regional concept, where APS estimation and compensation will be a fundamental processing step. In any case note that APS characteristics in the considered GB-RADAR data are quite severe due to the very short wavelength and the fact that the wave propagation occurs entirely within the lower part of the troposphere where APS fluctuations are more relevant. For this reason the APS conditions for the GESOAR systems are expected to be more favorable.

Fig. 8 (top) shows the coherence matrix calculated for a rural portion of the Bern scenario. A drop of the coherence in the day-time, in agreement with the $r$ parameter measurements reported above, can be observed. The coherence drop is much higher than the expected behavior according to Billingsley measurement campaigns for small wind conditions (from available weather information the wind was weak during the whole acquisition), indicated by the red box on the colorbar of the image. The red-box values have been derived according to the asymptotic value of the cross correlation derived by inverse transforming the Billingsley spectrum for $\gamma(t \rightarrow \infty)$ (see Appendix)

$$
\gamma=\gamma_{0} \frac{r}{1+r}
$$

where $\gamma_{0}$ is the maximum scene coherence limited by all decorrelation contributions (thermal noise, volumetric decorrelation, ...). The drop in the coherence is probably due to the rise of 

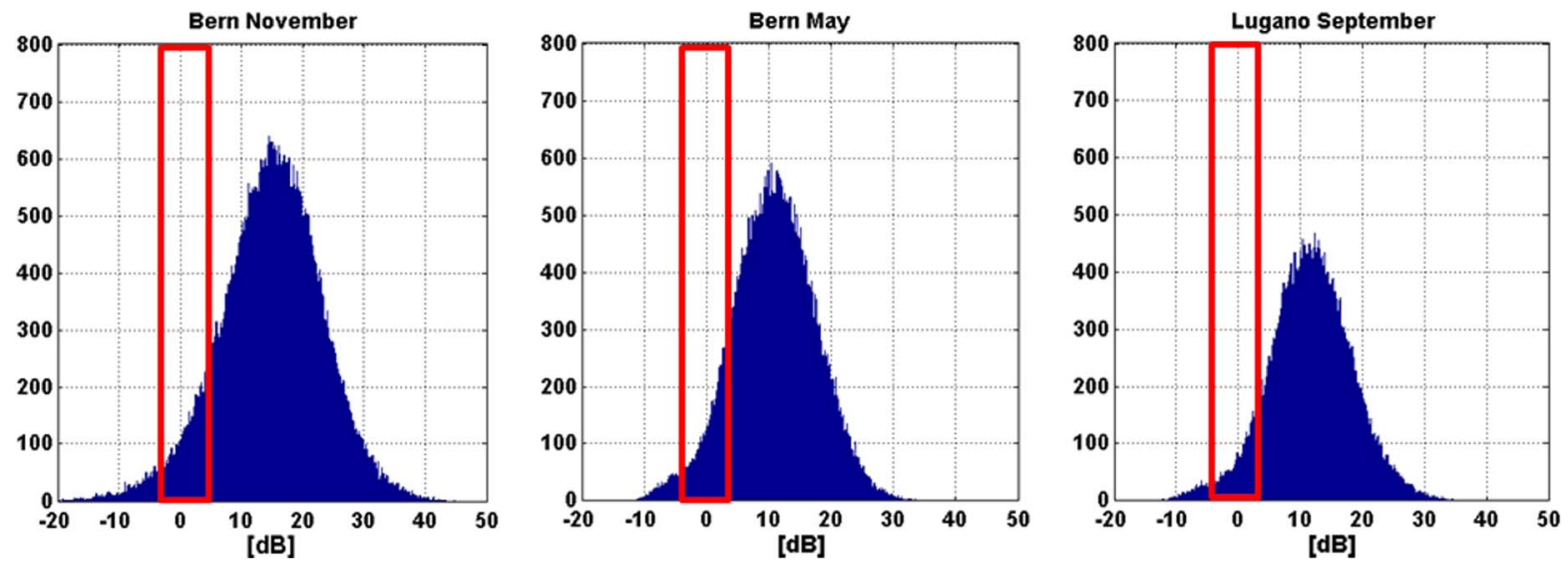

Fig. 6. Distribution of $r$ parameter estimated from GB-RADAR data over different sites and periods (Left: Bern November; center: Bern May; right: Lugano September). The red boxes indicate the range of parameter values observed during Billingsley measurement campaigns for small wind conditions.

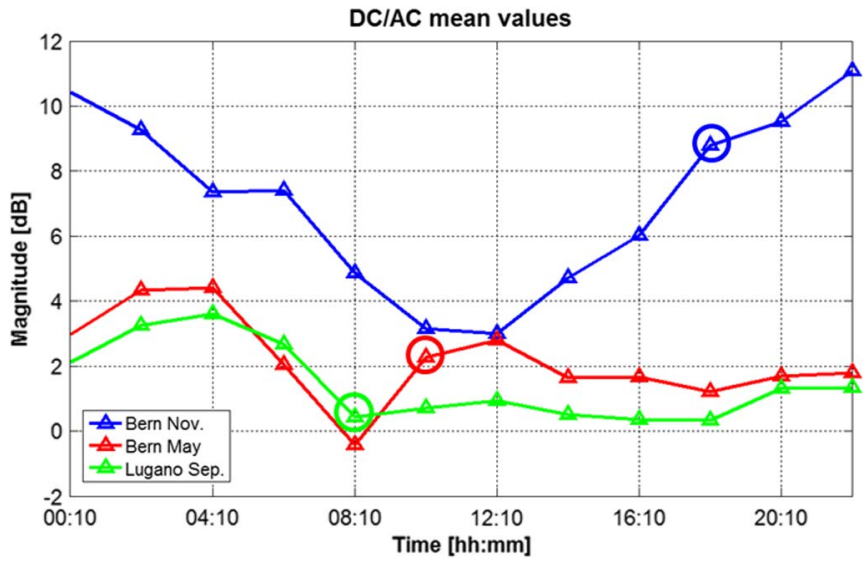

Fig. 7. Evolution of $r$ parameter versus time estimated from GB-RADAR data over different sites and periods (Blue: Bern November; red: Bern May; green: Lugano September). The staring time of each 24 -h campaign is indicated by the corresponding arrow.

the sun on the scene, warming and drying the surface of the targets, and locally changing the humidity of the air [24], [25].

Fig. 8 (bottom) shows the coherence variation w.r.t. time during the night (blue line) and during the day (red line). The decorrelation process clearly shows two different time constants, represented by the intersection of the dotted lines in the plot (initial coherence transition) with 0. During the night the coherence slowly decreases and would require more than $24 \mathrm{~h}$ to drop to 0 . This is not possible since at dawn the characteristics of the scene suddenly change (abrupt transition in the coherence matrix around 8 A.M.). During the day the coherence decrease is much faster and the time constant is less than $2 \mathrm{~h}$.

The different day/night characteristics of the imaged scene shall be considered when planning the acquisitions of a GEOSAR system. In particular, the night acquisitions should be preferred to obtain better quality images. Of course GEOSAR imaging during daytime is still possible during winter and for areas with cloud cover or located at high latitudes where sun effect is less relevant. In Appendix we provide a comparison between the short term decorrelation model proposed by Billingsley and the long-term decorrelation models considered for space-born SAR acquisitions.
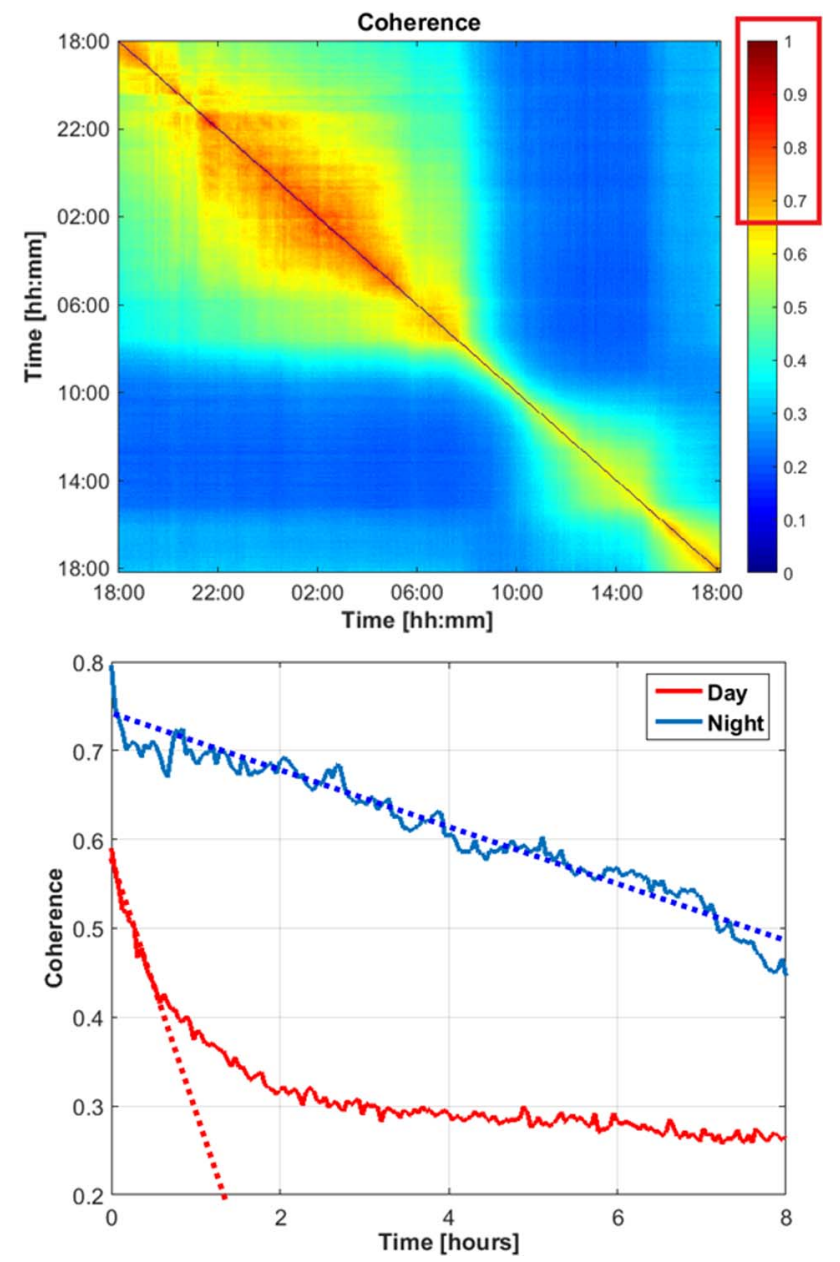

Fig. 8. (Top) 24-h coherence matrix for a rural area after APS compensation obtained from a GB RADAR campaign close to Bern. The red box on the colorbar of the image on the right indicates the range of expected coherence values according to Billingsley measurement campaigns for small wind conditions. (Bottom) Night (blue line) and day (red line) coherence variation versus time. The dotted lines represent the linear fit of the initial transitory of the coherence.

\section{GEOSAR Signal-to-Clutter Ratio Model}

\section{A. SCR Model in Focused GEOSAR Image}

We want to define a model for the azimuth spreading of the decorrelating clutter energy in a focused GEOSAR image. 

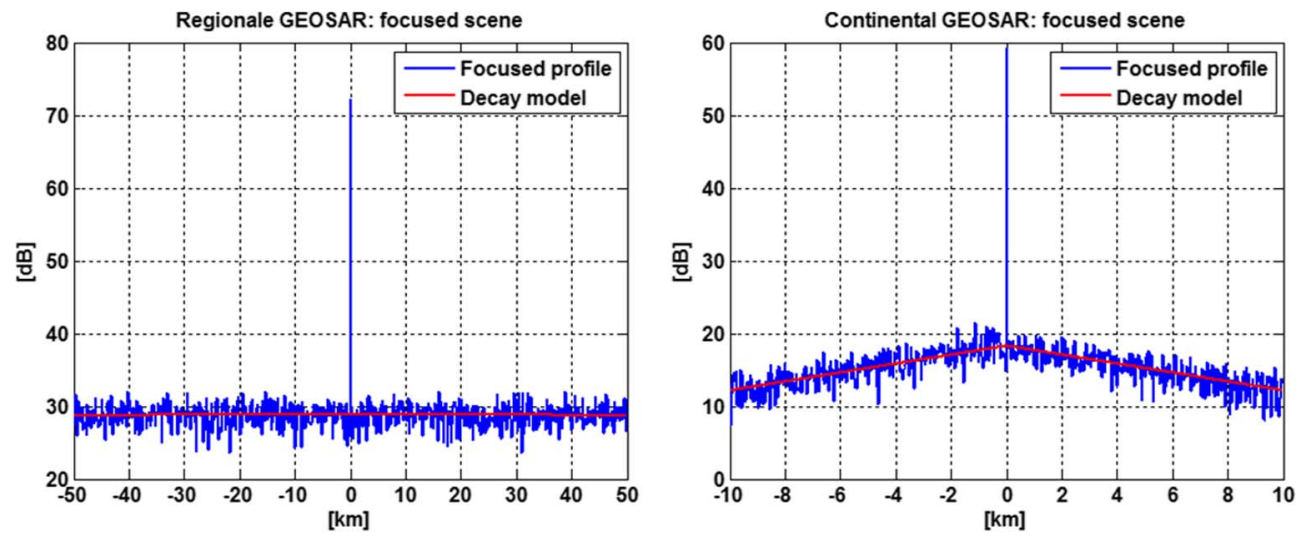

Fig. 9. Average azimuth profile of a set of simulated Billingsley-like targets after azimuth compression (blue line) and fit with the energy decay model (red) for a regional (left) and a continental (right) GEOSAR system.

The decorrelating clutter is a random process and the expected value of the clutter energy throughout the focused scene can be evaluated from (11) as

$$
E_{c}\left(f_{d}\right)=E\left\{\left|\widehat{s}\left(f_{d}\right)\right|^{2}\right\}=T S_{u}\left(f_{d}\right)
$$

where $S_{u}$ is the power spectrum of the random process modeling the clutter. We can now substitute the ICM power spectrum model in (22) and write the expected amount of energy within the focused image for a generic Billingsley-like target located at $y_{0}$

$$
E_{c}\left(y-y_{0}\right)=E_{d c}\left(y-y_{0}\right)+E_{a c}\left(y-y_{0}\right)
$$

where

$$
\begin{aligned}
& E_{d c}(\Delta y)=\frac{r}{r+1}\left|W\left(\frac{2 v \Delta y}{R_{0} \lambda}\right)\right|^{2} \\
& E_{a c}(\Delta y)=\frac{T}{(r+1)}\left[\frac{\beta \lambda}{4} \exp \left(-\frac{v \beta|\Delta y|}{R_{0}}\right)+C_{\text {alias }}\right] .
\end{aligned}
$$

We have defined the azimuth distance $\Delta y=y-y_{0}$ and the term $C_{\text {alias }}$, accounting for the clutter power at high frequencies folding back in base band due to the sampled SAR acquisition (fast decorrelating components in Fig. 3). In particular, the total power folding back, after range compression, is included between PRF and the inverse of the pulse length $T_{C}$

$$
\begin{aligned}
C_{\text {alias }}= & \frac{2 \int_{\mathrm{PRF} / 2}^{1 / 2 T_{C}} S_{u}\left(f_{d}\right) d f_{d}}{f_{\mathrm{PRF}}} \\
= & \frac{\exp \left(-\frac{\beta \lambda f_{\mathrm{PRF}}}{4}\right)-\exp \left(-\frac{\beta \lambda}{4 T_{C}}\right)}{f_{\mathrm{PRF}}} .
\end{aligned}
$$

Note that the higher the system duty cycle, the lower the alias level since $T_{C} \rightarrow 1 / f_{\mathrm{PRF}}$. Fig. 9 shows the average azimuth energy profile for a set of simulated Billingsley-like targets (blue line) compared with the provided decay model (red line). The plot on the left refers to a regional GEOSAR system $(T=900 \mathrm{~s}, \mathrm{PRF}=10 \mathrm{~Hz} v=5 \mathrm{~m} / \mathrm{s})$ while the plot on the right refers to a continental GEOSAR system $(T=20 \mathrm{~s}, \mathrm{PRF}=$ $150 \mathrm{~Hz} v=1500 \mathrm{~m} / \mathrm{s})$. X-band systems were considered in moderate wind conditions according to (20) and Table II ( $w=$ $10 \mathrm{~m} / \mathrm{s}, r=-6 \mathrm{~dB}, \beta=5.5)$. Note that due to the different acquisition parameters the energy profile is almost flat for the regional system whereas a decay rate of about $0.5 \mathrm{~dB} / \mathrm{km}$ can be observed for the continental system.

Billingsley ICM model can be exploited to evaluate the expected performances of a GEOSAR system in terms of SCR, defined as the ratio between the power of the coherent signal and the power of the decorrelating clutter within the focused scene. The decorrelating clutter power in a generic position of the focused scene is the integral over the whole antenna azimuth beam footprint $D$ of the stochastic part of the clutter power spectrum

$$
P_{C}=\rho_{r g} \int_{D} \sigma^{0}(\Delta y) E_{a c}(\Delta y ; r(\Delta y), \beta(\Delta y)) d \Delta y
$$

where $\rho_{r g}$ is the range resolution cell and the clutter parameters $\left(\sigma^{0}, r, \beta\right)$ depend on the azimuth location. The above equation is quite general and, provided that we have a complete characterization of the clutter of the imaged scene, would enable retrieving the expected clutter power for every pixel of the focused image. This is of course not possible for real scenarios even though, exploiting land classification maps and available radiometric information from other missions, an approximate estimation of expected decorrelating clutter nuisance could be obtained. In this paper, we define simplified scene models for a preliminary evaluation of the expected GEOSAR performances.

\section{B. Homogeneous Scenario}

The easiest model we assume is a distributed scenario made of homogeneous clutter in terms of both normalized radar cross section $\left(\sigma^{0}\right)$ and Billingsley model parameters $(r$ and $\beta)$. The coherent signal level is the integral over the azimuth resolution cell of the deterministic part of the clutter power spectrum

$$
P_{S}=\sigma^{0} \rho_{r g} \int_{\rho_{a z}} E_{d c}(\Delta y) d \Delta y=\sigma^{0} \rho_{r g} \rho_{a z} \frac{r}{r+1} T^{2} .
$$




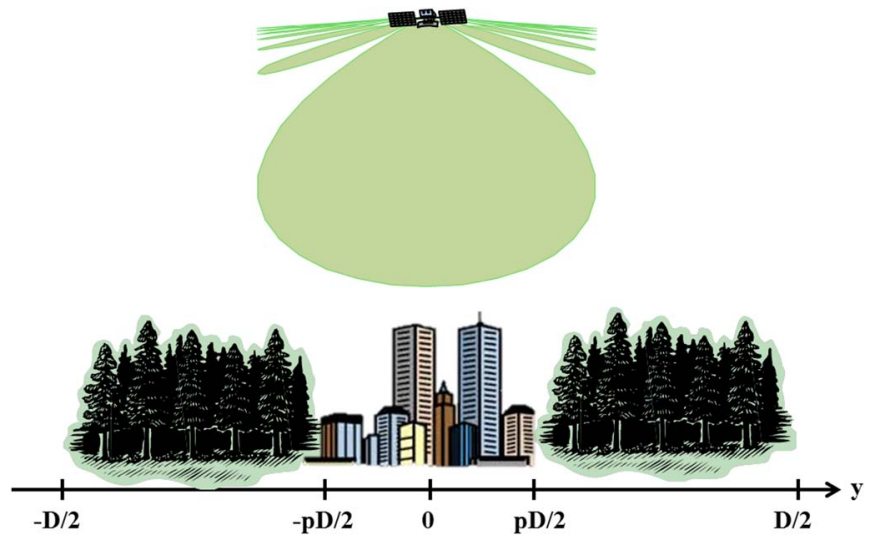

Fig. 10. Nonhomogeneous clutter scenario for SCR evaluation.

The clutter level evaluated at the center of the focused scene is the integral of the stochastic part of the clutter power spectrum over the antenna azimuth footprint $D$

$$
\begin{aligned}
P_{C} & =\sigma^{0} \rho_{r g} \int_{D} E_{a c}(\Delta y) d \Delta y \\
& =\sigma^{0} \rho_{r g} \frac{T}{r+1} \int_{D}\left[\frac{\beta \lambda}{4} \exp \left(-\frac{v \beta}{R_{0}}|\Delta y|\right)+C_{\text {alias }}\right] d \Delta y \\
& =\sigma^{0} \rho_{r g} \frac{T}{r+1}\left\{\frac{R_{0} \lambda}{2 v}\left[1-\exp \left(-\frac{v \beta D}{2 R_{0}}\right)\right]+D C_{\text {alias }}\right\} .
\end{aligned}
$$

The expected SCR is then

$$
\begin{aligned}
\mathrm{SCR} & =\frac{P_{S}}{P_{C}}=\frac{\rho_{a z} r T}{\frac{R_{0} \lambda}{2 v}\left[1-\exp \left(-\frac{v \beta D}{2 R_{0}}\right)\right]}+D C_{\text {alias }} \\
& =\frac{r}{1-\exp \left(-\frac{v \beta \lambda}{2 L_{\text {ant }}}\right)+\frac{2 v}{L_{\text {ant }}} C_{\text {alias }}}
\end{aligned}
$$

where we substituted $\rho_{a z}=\lambda /(2 v T) R_{0}$ and $D=\left(\lambda / L_{\text {ant }}\right) R_{0}$, where $L_{\text {ant }}$ is the equivalent antenna length.

\section{Nonhomogeneous Scenario}

The case of a nonhomogeneous scenario is also to be considered. The energy of decorrelating clutter, spreading through the focused image, is a further noise source even for stable targets. We assume here the worst case scenario, pictorially illustrated in Fig. 10, made of a region of stable targets (e.g., a city) at the center of the antenna beam surrounded by decorrelating clutter (e.g., a forest). The power of the coherent distributed targets is

$$
P_{S}=\rho_{r g} \rho_{a z} \sigma_{t}^{0} T^{2}
$$

where $\sigma_{t}^{0}$ is the NRCS of the coherent target. The clutter power level at the center of the stable region whose extent is a fraction $\alpha(0 \geq \alpha \geq 1)$ of the antenna beam width $D$ is

$$
P_{C}=2 \sigma_{c}^{0} \rho_{r g} \int_{\alpha D}^{D} E_{a c}(\Delta y) d \Delta y
$$

where $\sigma_{c}^{0}$ is the NRCS of the decorrelating clutter. After some algebra the inverse of the SCR can be written as

$$
\begin{aligned}
& \mathrm{SCR}^{-1}=\frac{P_{C}}{P_{S}}=\frac{\sigma_{c}^{0}}{\sigma_{t}^{0}(r+1)} \frac{2 v(1-\alpha)}{L_{\mathrm{ant}}} C_{\text {alias }} \\
& +\frac{\sigma_{c}^{0}}{\sigma_{t}^{0}(r+1)}\left[\exp \left(-\frac{v \beta \alpha \lambda}{2 L_{\mathrm{ant}}}\right)-\exp \left(-\frac{v \beta \lambda}{2 L_{\mathrm{ant}}}\right)\right] .
\end{aligned}
$$

By approximating the exponential terms with the Taylor expansion we can rewrite (33) in a compact way

$$
\mathrm{SCR}^{-1}=\frac{\sigma_{c}^{0}}{\sigma_{t}^{0}} \frac{(1-\alpha)\left(\frac{\beta \lambda}{4}+C_{\mathrm{alias}}\right)}{r+1} \frac{2 v}{L_{\mathrm{ant}}} .
$$

Three terms can be noticed: the first represents the contrast between the reflectivity of clutter and targets, the second depends on the nature of the clutter and the third is system dependent. The previous expression allows to understand which is the most critical nuisance for a GEOSAR system between thermal noise and clutter. In particular, in case the SCR (calculated for $\sigma_{t}^{0}$ equal the system Noise Equivalent Sigma Zero) is lower than 1 the system is clutter limited.

\section{GEOSAR CASE STUdies}

The SCR models from Section IV allow to derive the expected performances for a GEOSAR system. In this section we provide a preliminary performance assessment for the main GEOSAR concepts. The calculations are based on the Billingsley ICM model for both the systems even though, as shown in Section III-B, there is little evidence that the model can be exploited as it is, especially for a TLCOMP system with very long integration times. Nevertheless, for a complete statistical characterization of the clutter decorrelation over long observation times the processing of large amounts of data acquired at different bands, seasons, weather conditions, and locations would be required. This task is outside the purpose of the present paper and the provided performances offer, in any case, a preliminary indication of the potential issues to be tackled during the design of a GEOSAR system.

\section{A. GEOSAR Continental System}

The integration time for a continental system goes from seconds to few minutes and the ICM model is a reasonable approximation of the decorrelating clutter. In particular, the main source of clutter will be the windblown trees since other classes of targets are expected to have higher decorrelation times. For such system we can also exploit the experimental relationships between wind and model parameters in (20) and Table II.

Fig. 11 shows the SCR evaluated from (30) as a function of wind speed and carrier frequency for a GEOSAR continental system $\left(v=2600 \mathrm{~m} / \mathrm{s}, f_{\mathrm{PRF}}=200 \mathrm{~Hz}, L_{\text {ant }}=20 \mathrm{~m}\right)$. For clarity the $0-\mathrm{dB}$ contour has been highlighted with the black line. The image refers to a homogeneous forest. Only at L-band the SCR is good for every wind condition. The SCR level can be improved by reducing the azimuth resolution of the image through pre-summing of the raw data (corresponding to a virtual increase of the antenna size). By imposing an objective 


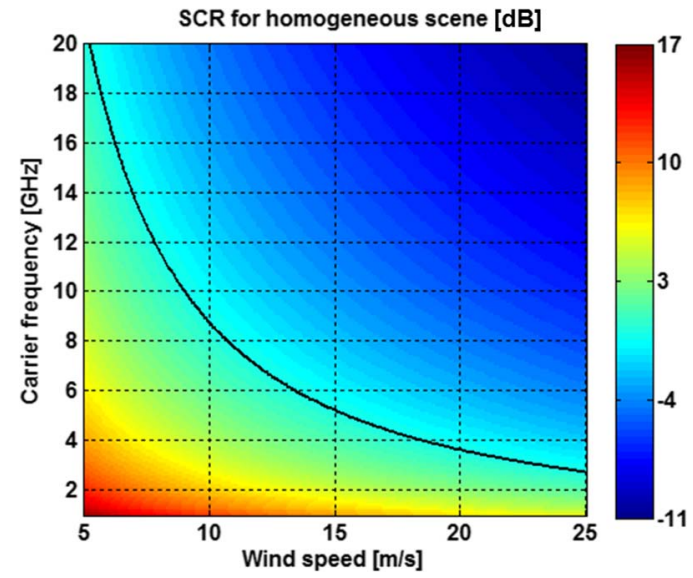

Fig. 11. Signal-to-clutter ratio for homogeneous scene for GEOSAR continental system as a function of wind speed and working band according to Billingsley ICM model for trees. The black line represents the 0 -dB contour.

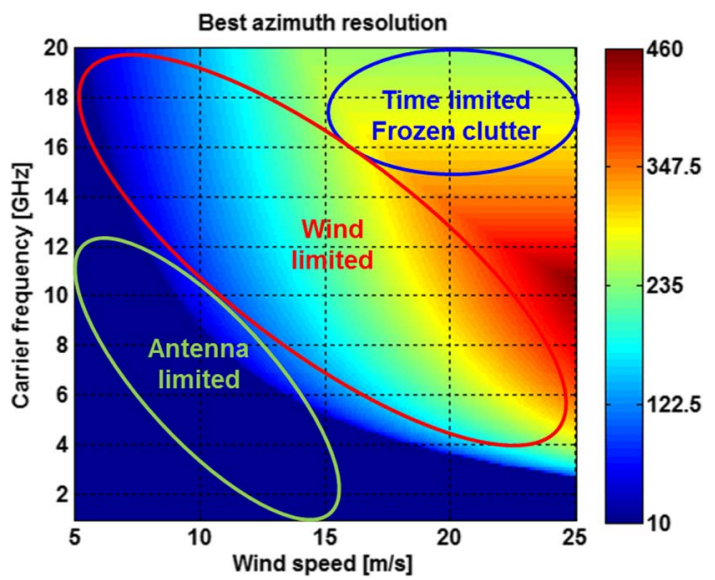

Fig. 12. Best azimuth resolution for a GEOSAR continental system over a forest as a function of wind velocity and carrier frequency.

SCR of $0 \mathrm{~dB}$ it is possible to derive the best possible resolution over a forest as

$$
\rho_{a z}=\min \left(-\frac{\lambda v \beta}{4 \log (1-r)}, \frac{\lambda \tau_{0} R_{0}}{2 v_{\text {ground }}}\right) .
$$

The first term of (35) has been derived from (30), neglecting the $C_{\text {alias }}$ term which is very small for a continental system due to the high PRF. The second term is a lower bound for $\rho_{a z}$ reached when the system integration time is of the same order of magnitude of the clutter decorrelation time $\tau_{0}$. Fig. 12 shows the best achievable azimuth resolution as a function of wind speed and carrier frequency. A clutter decorrelation time of about $3 \mathrm{~s}$ was assumed. Three different behaviors can be noticed:

- Antenna-limited resolution: The resolution does not depend on wind speed but on the physical dimension of the antenna $\rho_{a z}=L_{\text {ant }} / 2$.

- Wind-limited resolution: The resolution deteriorates at the increase of wind speed

- Time-limited resolution: The resolution does not depend on wind speed but on the clutter decorrelation time. In this case the frozen clutter assumption is valid and the standard clutter models [8] can be applied.

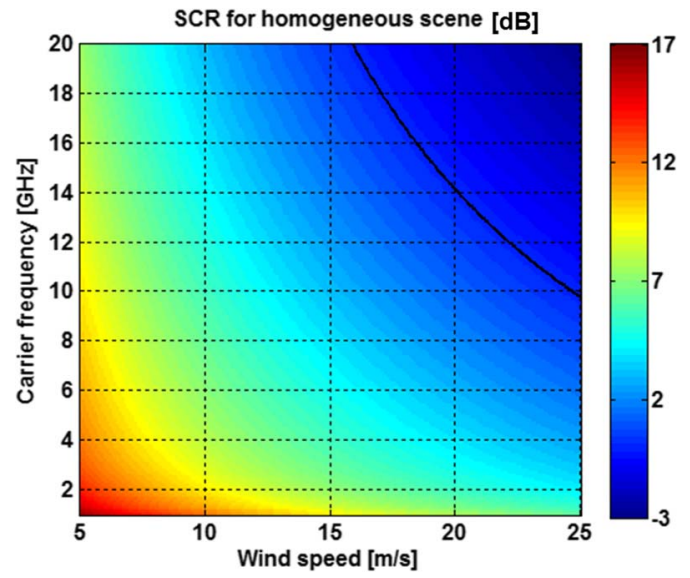

Fig. 13. Signal-to-clutter ratio for homogeneous scene for GEOSAR regional system as a function of wind speed and working band according to Billingsley ICM model for trees. The black line represents the $0 \mathrm{~dB}$ contour.

\section{B. GEOSAR Regional System}

The regional GEOSAR system is characterized by long integration times from several minutes to hours. The applicability of Billingsley ICM model is then subject to the stationarity of the decorrelation process which in turn will depend on the acquisition conditions (e.g., weather, time of the day, ...). Furthermore, in such long integration time even targets different from trees (grass, cultivated fields, ...) can decorrelate introducing further sources of noise. The results shown in this section provide in any case a preliminary assessment of the performances of a regional GEOSAR system in the presence of clutter decorrelation. Fig. 13 shows the SCR evaluated from (30) as a function of wind speed and carrier frequency for a GEOSAR regional system $\left(v=5 \mathrm{~m} / \mathrm{s}, f_{\mathrm{PRF}}=35 \mathrm{~Hz}\right.$, $L_{\text {ant }}=3 \mathrm{~m}$ ). For clarity the $0-\mathrm{dB}$ contour has been highlighted with the black line. The image refers to a homogeneous scene of wind-blown trees. The performances are better than the continental case since only for very strong wind conditions the $\mathrm{SCR}$ is lower than 0 for carrier frequencies higher than $10 \mathrm{GHz}$. The acquisition configuration of the regional GEOSAR system is indeed favorable to clutter noise mitigation thanks to the small ratio between targets and system Doppler bandwidths, resulting in part of the clutter energy spreading outside the focused scene.

The performances of Fig. 13 should improve for a nonhomogeneous scenario where a certain fraction of permanent scatterers is present. To assess the effects of clutter over a real scenario we performed the reconditioning of LEOSAR data into a regional GEOSAR demo product. The original data set was a stack of COSMO-SkyMed data over Milano (X-band) which was exploited to identify the PS within the scene. All other targets were assumed to decorrelate during the long integration time. In particular, a fraction of the power of such targets was not changed (dc part), and the remaining fraction of power was spread through the focused image (ac part). The dc/ac ratio was evaluated according to (20) for a wind speed of $5 \mathrm{~m} / \mathrm{s}$ corresponding to $r=-3.6 \mathrm{~dB}$. According to the available wind statistics for Milano Linate (derived for power generation purposes), the wind speed in Milano is for the $95 \%$ of the time lower than the considered velocity. 


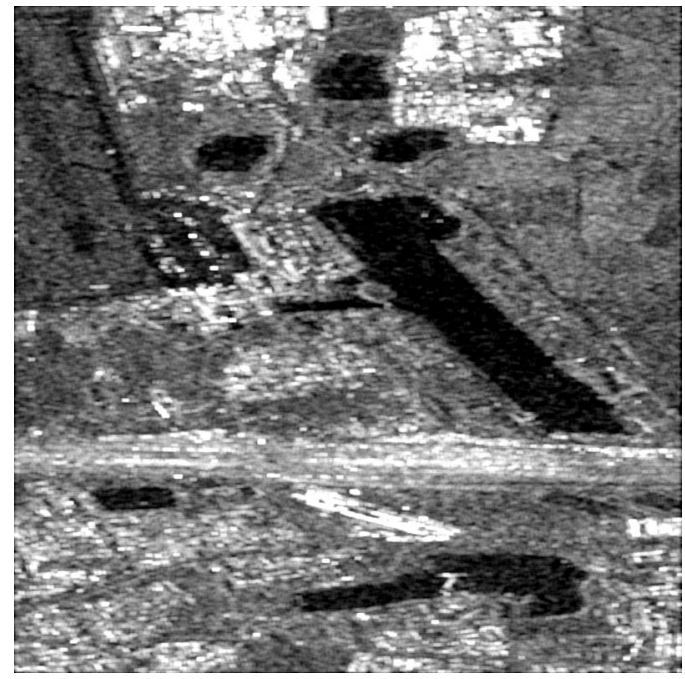

Fig. 14. Detail of the original COSMO-SkyMed image over Milano after resolution change and multi-looking (ML factor 50).

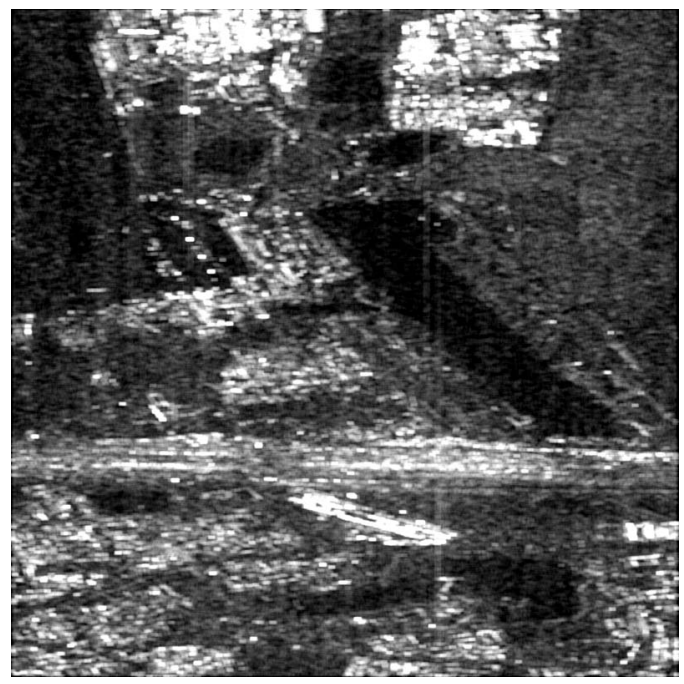

Fig. 15. Detail of the reconditioned GEOSAR demo product after clutter decorrelation (ML factor 50).

Fig. 14 shows a detail of the original COSMO-SkyMed product used for the reconditioning operation. The performed reconditioning steps have been: the change of resolution according to the expected GEOSAR values $\left(\rho_{a z}=10 \mathrm{~m}, \rho_{r g}=\right.$ $5 \mathrm{~m}$ ), the separation of PS from non stable targets exploiting the stack of original LEOSAR data, and finally the introduction of the clutter decorrelation according to Billingsley ICM model parameters. In particular, the final image has been obtained as the sum of three different components: the PS component unmodified by the processing, the stable clutter component reduced of a fraction of power proportional to Billingsley dc part and the decorrelating clutter reduced of a fraction of power proportional to Billingsley ac part and affected by decorrelation. The decorrelation was introduced by applying to the signal spectrum a phase noise with the same spectral characteristics of ICM model. Fig. 15 shows the results of the processing. The radiometric quality of the GEOSAR demo product is clearly worse than the original COSMO-SkyMed product. The impact of the clutter decorrelation on the image was evaluated by

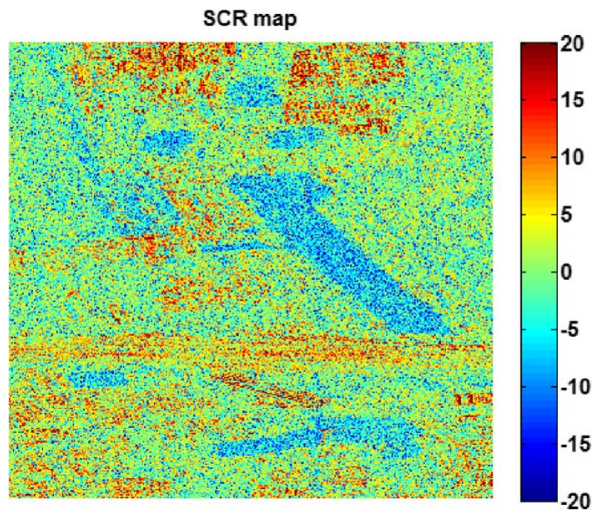

Fig. 16. Signal-to-clutter-ratio map for the reconditioned GEOSAR demo product.

calculating the SCR map, represented in Fig. 16. Most of the SCR values are included in the range $0-5 \mathrm{~dB}$ but lower values can be found for particularly dark areas while higher values indicate the presence of very bright man-made urban targets.

\section{CONCLUSION}

The paper analyzes the issue of scene decorrelation for geosynchronous SAR systems exploiting long integration times to achieve high resolution and good SNR. The decorrelating clutter energy spreads throughout the focused scene impacting the quality of the focused images. We have derived a theoretical model for the clutter energy spreading based upon the power spectrum of the stochastic process representing the decorrelation.

The Billingsley Intrinsic Clutter Motion model is a widely accepted way to represent the clutter power spectrum with an exponential decay of the clutter energy. By exploiting such model we have derived the expected performances for a GEOSAR system in terms of SCR. The provided models can be exploited to design GEOSAR systems robust in front of the clutter decorrelation issue. Furthermore, we have presented the preliminary results from a set of GB-RADAR measurement campaigns aimed at extending the validity of ICM model, which is mainly valid for short observation times and for windblown trees. The preliminary analysis shows exponential decay rates much higher than those measured by Billingsley. Furthermore, we observed that, at least in Ku band, an important source of decorrelation for almost every target is the sun. The acquisitions performed during the day show dc/ac ratio values lower (up to $15 \mathrm{~dB}$ ) than those measured during night acquisitions. This would lead to prefer night acquisitions for a GEOSAR system working in $\mathrm{X}$ or Ku band, at least during summer period and for midlatitude regions.

The derived performance models were finally evaluated for two different GEOSAR concepts. The continental system characterized by high sensor velocity could experience some problems in imaging densely vegetated areas. Only reducing azimuth resolution (by pre-summing of raw data) would allow to obtain good quality images. On the contrary the regional GEOSAR system is more robust to clutter decorrelation thanks to the reduced sensor velocity, resulting in a small scene bandwidth with respect to the system PRF. In any case further 
analysis on the long-term clutter statistics are required for the TLCOMP GEOSAR concept in order to properly account for the long integration time required by this system.

\section{APPENDiX A \\ TARgets Decorrelation Model COMParison}

The coherence between two SAR data $x(t)$ separated by a time interval $\Delta$ is defined as

$$
\begin{aligned}
\gamma & =\frac{E\left[x(t) x^{*}(t+\Delta)\right]}{\sqrt{E\left[|x(t)|^{2}\left|x^{*}(t+\Delta)\right|\right]}} \\
& =\frac{E\left[x(t) x^{*}(t+\Delta)\right]}{P_{x}}=\frac{r_{x}(\Delta)}{r_{x}(0)} .
\end{aligned}
$$

This expression can be related to the ICM model by evaluating the autocorrelation of the clutter power spectral density (18)

$$
r_{x}(t)=\frac{1}{r+1} \frac{1}{1+\left(\frac{4 \pi t}{\lambda \beta}\right)^{2}}+\frac{r}{r+1} .
$$

We can now express the coherence as a function of the the parameters of the ICM model

$$
\gamma(\Delta)=\frac{1}{r+1}\left(\frac{1}{1+\left(\frac{4 \pi \Delta}{\lambda \beta}\right)^{2}}+r\right)
$$

where, as expected, the coherence is unitary for $\Delta=0$ while $\gamma(\Delta \rightarrow \inf )=r /(r+1)$. The dc/ac parameter defines the long-term coherence, whereas the $\beta$ parameter describes the short term behavior. The long-term coherence will be affected by acquisition condition changes (e.g., wind or day/night).

The dc/ac long-term variations can be described exploiting the models usually assumed for SAR acquisitions. The first model, valid for long observation times, is [26]

$$
\begin{aligned}
\gamma(\Delta t) & =\exp \left(-\frac{\Delta t}{\tau^{2}}\right) \\
\tau & =\frac{\lambda}{4 \pi} \frac{\sqrt{2}}{\sigma_{d}}
\end{aligned}
$$

derived assuming Brownian motion, that is the displacements randomly change with stationary Gaussian increments distributed as $N\left(0, \sigma_{d}\right)$. A second model, similar to the first but assuming stationary Brownian motion in increments of velocity (acceleration) is [27]

$$
\begin{aligned}
\gamma(\Delta t) & =\exp \left(-\left(\frac{\Delta t}{\tau}\right)^{2}\right) \\
\tau & =\frac{\lambda}{4 \pi} \frac{\sqrt{2}}{\sigma_{v}}
\end{aligned}
$$

where $\sigma_{v}$ is the standard deviation of the velocity increments.

Please note that the models have been empirically derived exploiting spaceborne stacks of interferometric images, with revisit time of days, whereas in GEOSAR we are interested in decorrelation times of hours. For this reason, the available GB-RADAR campaigns should be further exploited to derive a model linking the short-term decorrelation described by Billingsley with the long-term models derived from spaceborne SAR.

\section{ACKNOWLEDGMENT}

Part of the activities described in the paper was carried out within the framework of the ESA-ESTEC funded project: Study on Utilization of Future Telecom Satellites for Earth Observation. The authors wish to thank T. Strozzi and C. Werner from GAMMA for performing the radar acquisitions presented in this paper. The authors also thank M. Belotti and D. Giudici from ARESYS for helping in the processing of the GB-RADAR data and in the generation of the GEOSAR demo products.

\section{REFERENCES}

[1] K. Tomiyasu, "Synthetic aperture radar in geosynchronous orbit," in Proc. Antennas Propag. Soc. Int. Symp., 1978, vol. 16, pp. 42-45.

[2] K. Tomiyasu and J. L. Pacelli, "Synthetic aperture radar imaging from an inclined geosynchronous orbit," IEEE Trans. Geosci. Remote Sens., vol. GE-21, no. 3, pp. 324-329, Jul. 1983.

[3] W. N. Edelstein, S. N. Madsen, A. Moussessian, and C. Chen, "Concepts and technologies for synthetic aperture radar from meo and geosynchronous orbits," in Proc. SPIE, 2005, vol. 5659, pp. 195-203.

[4] D. Bruno and S. Hobbs, "Radar imaging from geosynchronous orbit: Temporal decorrelation aspects," IEEE Trans. Geosci. Remote Sens., vol. 48, no. 7, pp. 2924-2929, Jul. 2010.

[5] J. Ruiz Rodon, A. Broquetas, A. Monti Guarnieri, and F. Rocca, "Geosynchronous SAR focusing with atmospheric phase screen retrieval and compensation," IEEE Trans. Geosci. Remote Sens., vol. 51, no. 8, pp. 4397-4404, Aug. 2013

[6] C. Hu, T. Long, T. Zeng, F. Liu, and Z. Liu, "The accurate focusing and resolution analysis method in geosynchronous SAR," IEEE Trans. Geosci. Remote Sens., vol. 49, no. 10, pp. 3548-3563, Oct. 2011.

[7] Y. Tian et al., "Theoretical analysis and verification of time variation of background ionosphere on geosynchronous SAR imaging," IEEE Geosci. Remote Sens. Lett., vol. 12, no. 4, pp. 721-725, Apr. 2015.

[8] F. T. Ulaby and M. C. Dobson, Handbook of Radar Scattering Statistics for Terrain. Dedham, MA, USA: Artech House, 1989.

[9] R. M. M. Walter, G. Carrara, and R. S. Goodman, Spotlight Synthetic Aperture Radar: Signal Processing Algorithms. Norwood, MA, USA: Artech House, 1995.

[10] M. Wicks, M. Rangaswamy, R. Adve, and T. Hale, "Space-time adaptive processing: A knowledge-based perspective for airborne radar," IEEE Signal Process. Mag., vol. 23, no. 1, pp. 51-65, Jan. 2006.

[11] D. Pastina and F. Turin, "Exploitation of the cosmo-skymed SAR system for gmti applications," IEEE J. Sel. Topics Appl. Earth Observ. Remote Sens., vol. 8, no. 3, pp. 966-979, Mar. 2015.

[12] J. Billingsley, Low-Angle Radar Land Clutter: Measurements and Empirical Models, ser. Radar, Sonar, Navigation and Avionics Bks. Norwich, NY, USA: William Andrew Publ., 2002. [Online]. Available: http://books. google.it/books?id=FEkn0-h7sz0C

[13] A. Recchia, A. Monti Guarnieri, A. Broquetas Ibars, and J. Ruiz Rodon, "Impact of clutter decorrelation on geosynchronous sar," in Proc. 10th EUSAR, Jun. 2014, pp. 1-4.

[14] J. Ruiz-Rodon, A. Broquetas, E. Makul, A. Monti-Guarnieri, and A. Recchia, "Internal clutter motion impact on the long integration GEOSAR acquisition," in Proc. IEEE IGARSS, Jul. 2014, pp. 2343-2346.

[15] C. Prati, F. Rocca, D. Giancola, and A. Guarnieri, "Passive geosynchronous SAR system reusing backscattered digital audio broadcasting signals," IEEE Trans. Geosci. Remote Sens., vol. 36, no. 6, pp. 1973-1976, Nov. 1998.

[16] S. Madsen, W. Edelstein, L. DiDomenico, and J. LaBrecque, "A geosynchronous synthetic aperture radar; for tectonic mapping, disaster management and measurements of vegetation and soil moisture," in Proc. IEEE IGARSS, 2001, vol. 1, pp. 447-449.

[17] A. Guarnieri, S. Tebaldini, F. Rocca, and A. Broquetas, "Gemini: Geosynchronous SAR for earth monitoring by interferometry and imaging," in Proc. IEEE IGARSS, 2012, pp. 210-213. 
[18] L. M. H. Ulander, H. Hellsten, and G. Stenstrom, "Synthetic-aperture radar processing using fast factorized back-projection," IEEE Trans. Aerosp. Electron. Syst., vol. 39, no. 3, pp. 760-776, Jul. 2003.

[19] A. Recchia, A. Monti Guarnieri, A. Broquetas, and J. Ruiz-Rodon, "Assesment of atmospheric phase screen impact on geosynchronous SAR," in Proc. IEEE IGARSS, Jul. 2014, pp. 2253-2256.

[20] P. Lombardo and J. B. Billingsley, "A new model for the Doppler spectrum of windblown radar ground clutter," in Rec. IEEE Radar Conf., 1999, pp. $142-147$.

[21] T. Strozzi, A. Wiesmann, and U. Wegmuller, "Gamma's portable radar interferometer," in Proc. Symp. Deform. Meas. Anal., Lisbon, Portugal, 2008, pp. 1-10.

[22] P. Lombardo, M. Greco, F. Gini, A. Farina, and J. Billingsley, "Impact of clutter spectra on radar performance prediction," IEEE Trans. Aerosp. Electron. Syst., vol. 37, no. 3, pp. 1022-1038, Jul. 2001.

[23] P. Guccione, A. Monti-Guarnieri, and S. Tebaldini, "Stable target detection and coherence estimation in interferometric SAR stacks," IEEE Trans. Geosci. Remote Sens., vol. 50, no. 8, pp. 3171-3178, Aug. 2012.

[24] L. Iannini and A. Guarnieri, "Atmospheric phase screen in ground-based radar: Statistics and compensation," IEEE Geosci. Remote Sens. Lett., vol. 8, no. 3, pp. 537-541, May 2011.

[25] F. De Zan, M. Zonno, P. López-Dekker, and A. Parizzi, "Phase inconsistencies and water effects in SAR interferometric stacks," in Proc. Fringe Workshop, 2015, pp. 1-30.

[26] F. Rocca, "Modeling interferogram stacks," IEEE Trans. Geosci. Remote Sens., vol. 45, no. 10, pp. 3289-3299, Oct. 2007.

[27] H. Zebker and J. Villasenor, "Decorrelation in interferometric radar echoes," IEEE Trans. Geosci. Remote Sens., vol. 30, no. 5, pp. 950-959, Sep. 1992.

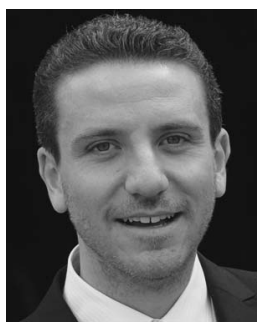

Andrea Recchia was born in Bergamo, Italy, on July 7,1983 . He received the master's degree in telecommunication engineering from Politecnico di Milano, Milano, Italy, in 2008, and the Ph.D. degree aimed at assessing the feasibility of a novel geosynchronous SAR system in the presence of scene and APS decorrelation, in 2015.

Since 2008 he joined Aresys, a PoliMI spin-off, specialized in radar and geophysics remote sensing solutions. He is currently part of the SAR R\&D team with particular interest in SAR data processing, SAR data quality assessment, and future missions design and evaluation. In 2009 he joined the Synthetic Aperture Radar study team at Dipartimento di Elettronica, Informazione e Bioingegneria, Politecnico di Milano.

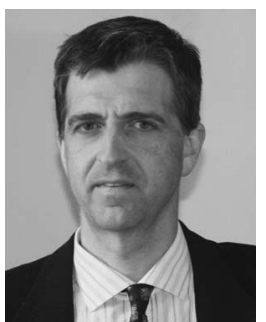

Andrea Monti Guarnieri (M'07) received the M.Sc. degree in electronic engineering from Politecnico de Milano, Milano, Italy, in 1988.

He had been an Assistant Professor and Full Professor habilitation within Dipartimento di Elettronica, Informazione e Bioingegneria, Politecnico de Milano. He has been teaching several courses on digital and statistical signal and image processing, telecommunications, and Radar. Almost 40 years of professional activities in SAR systems design and processing led him in 2003 to found Aresys, a PoliMI spin-off, specialized in radar and geophysics remote sensing solutions, then he served as the President up to 2015. His current interests focus on processing and calibration of ground, airborne and spaceborne SAR, multi-baseline interferometric and MIMO configurations, and geosynchronous SAR.

Prof. Monti Guarnieri coauthored over 200 scientific publications, of which 45 international peer-reviewed publications, (H-Index 23, 2600 citations); he was awarded three Best Paper in international symposia (IGARSS '89, EUSAR 2004, EUSAR 2012), and he is a coauthor of four patents. He is a reviewer of journals in remote sensing, signal processing, image processing, geophysics, geodynamics, and antennas and propagations, and he has been in many technical boards of SAR and RADAR conferences. In 2014 he was appointed by the board of directors member of Technical-scientific Committee of Italian Space Agency (ASI).

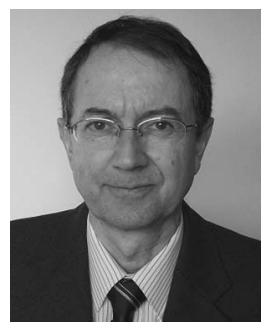

Antoni Broquetas (S'84-M'90) was born in Barcelona, Spain, in 1959. He received the Ingeniero degree in telecommunication engineering from the Universitat Politecnica de Catalunya (UPC), in 1985 and the Doctor Ingeniero degree in telecommunications engineering for his work on microwave tomography from UPC, in 1989.

In 1986 he was a Research Assistant in the Portsmouth Polytechnic (U.K.) involved in propagation studies. In 1987 he joined the Department of Signal Theory and Communications of the School of Telecommunication Engineering of the UPC in Barcelona.

In 1991 he started the remote sensing research activities at UPC working on the interferometric applications of space-borne synthetic aperture radars. From 1998 to 2002 he was a Subdirector of Research at the Institute of Geomatics in Barcelona. From 1999 he is a Full Professor in the UPC involved in research on radar imaging and remote sensing. From 2003 to 2006 he was the Director of the Signal Theory and Communications Department at UPC. He has published more than 170 papers on microwave tomography, Radar, ISAR and SAR systems, SAR processing, and interferometry.

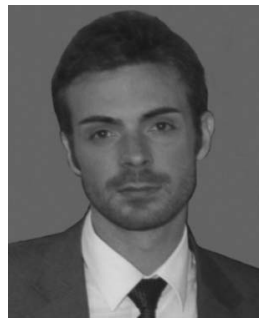

Antonio Leanza received the M.Sc. degree in telecommunication engineering from Politecnico di Milano, Milano, Italy, in 2013. He is currently pursuing the Ph.D. degree in information technology from Politecnico di Milano.

He worked for the remote sensing company Aresys from 2013 to 2014. His activities focus on synthetic aperture radar and particularly on the analysis of novel geosynchronous SAR systems for terrain and atmosphere observation. 